\title{
Lattice study on chiral dynamics of two-color six-flavors QCD
}

\section{Masaaki Tomii*}

High Energy Accelerator Organization(KEK), Tsukuba 305-0801, Japan

School of High Energy Accelerator Science, Graduate University for Advanced Studies

(Sokendai), Tsukuba 305-0801, Japan

\section{Masashi Hayakawa}

Department of Physics, Nagoya University, Nagoya 464-8602, Japan

\section{Ken-Ichi Ishikawa}

Department of Physics, Hiroshima University, Higashi-Hiroshima 739-8526, Japan

\section{Shinji Takeda}

School of Mathematics and Physics, College of Science and Engineering, Kanazawa University, Kanazawa 920-1192, Japan

\section{Norikazu Yamada}

High Energy Accelerator Organization(KEK), Tsukuba 305-0801, Japan

School of High Energy Accelerator Science, Graduate University for Advanced Studies

(Sokendai), Tsukuba 305-0801, Japan

E-mail: tomiiepost.kek.jp

\begin{abstract}
The electroweak symmetry breaking and origin of masses may be attributed to the breakdown of chiral symmetry due to a strong gauge dynamics. Among several candidates of such gauge systems, we focus on two-color QCD with $N_{f}=6$ massless Dirac fermions in the fundamental representation, and study on whether the dynamics of this gauge system trigger chiral symmetry breaking or not by simulating with Wilson fermions on lattices up to $L / a=32$. We show the result for the quark mass dependence and the volume dependence of some quantities such as the mass of the lightest pseudoscalar meson, decay constant, and give the three evidences supporting the absence of chiral symmetry breaking in the six-flavor theory.
\end{abstract}

31st International Symposium on Lattice Field Theory - LATTICE 2013

July 29 - August 3, 2013

Mainz, Germany

\footnotetext{
*Speaker.
} 


\section{Introduction}

The purpose of this study is to find a gauge system which may cause the electroweak (EW) symmetry breaking. Nowadays, we know that such gauge dynamics must give rise to large anomalous dimension $(\sim 1)$ over wide range of energy scale and also need the spontaneous breakdown of chiral symmetry at lower scale.

We focus here on a series of $\mathrm{SU}(2)_{C}$ gauge theories. As for $\mathrm{SU}(2)_{C}$ gauge theories, the system with two adjoint Dirac fermions has been studied extensively using numerical methods since the first work [W]. More recent studies of running coupling constant calculation [0, B] and scaling of the spectrum [四] suggest that this system has an IR-fixed point.

We investigate $\mathrm{SU}(2)_{\mathrm{C}}$ gauge theories with $N_{f}$ Dirac fermions in the fundamental representation. $\mathrm{SU}(2)_{\mathrm{C}}$ gauge theory is one of the $\mathrm{Sp}(N)$ gauge theories so that its fundamental representation is pseudoreal and that the plausible pattern of chiral symmetry breaking is $\mathrm{SU}\left(2 N_{f}\right) \rightarrow \operatorname{Sp}\left(2 N_{f}\right)$. Therefore the chiral dynamics of two-color QCD is different from that of three-color QCD. From the point of view of the application to the dynamical realization of EW symmetry breaking, the effective Higgs sector of $\mathrm{SU}(2)_{C}$ gauge system is quite different from that of $\mathrm{SU}(3)_{\mathrm{C}}$ gauge system. This fact also motivates us to perform lattice simulations to grasp its properties of nonperturbative dynamics such as spectra of bound states.

Study of the system with fundamental fermions intended to search the conformal window has also been done. The perturbative approach [5] suggests $6 \leq N_{f}^{\text {crtl }} \leq 8$. Reference [ $[$ ] investigated the phase structure of Wilson fermions and indicated $N_{f}=3$ system is conformal in the IR limit. Afterwards, running gauge coupling constant has been calculated nonperturbatively for two-color QCD and then the conformal signal was indicated for the system with $N_{f}=6$ [ $[\mathrm{U}], N_{f}=8$ [ [8], and $N_{f}=10$ [Q], while the QCD-like signal was indicated for the system with $N_{f}=4$ by Ref. [Q].

We focus on the chiral properties of $\mathrm{SU}(2)_{\mathrm{C}}$ gauge theory with six Dirac fermions in the fundamental representation and show the result for the quark mass dependence and the volume dependence of some quantities, such as the mass of the lightest pseudoscalar meson, and decay constant. Here we especially pay attention to the finite size effect on these quantities. We carefully make sure which data do not suffer the finite size effect to extract data which give the values in the infinite volume system and then compare these results with the predictions from the theory with the breakdown of chiral symmetry (Sec. \, 耳). In some cases, we conversely use the finite size effect that should reflect the long distance dynamics, and compare the obtained data with the expectations in the system with the breakdown of chiral symmetry (Sec. [1]).

In this research, we employ the plaquette gauge action and the Wilson fermion action on the lattices where spacial sizes are 16,24 , and 32 . In order to see the phase structure as a statistical system and fix a parameter $\beta$, which is inversely proportional to the square of the bare coupling, we investigate the hopping parameter dependence of the plaquette for several $\beta$. We choose $\beta=2.0$ where the bulk phase transition does not seem to occur.

In the following sections, we give three signatures supporting that the six-flavor theory is not the one with chiral symmetry breaking. Here we report the essential points of them. The full qualitative discussion is given in the full paper [10]]. 


\section{Pseudoscalar Spectrum}

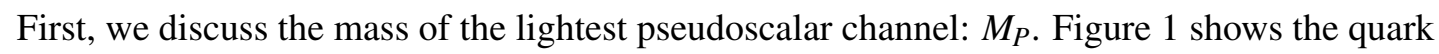
mass dependence of $M_{P} . M_{P}$ in $L / a=16$ and that in $L / a=24$ are consistent at $a m_{\mathrm{PCAC}}>0.2$. For smaller $m_{\mathrm{PCAC}}$, on the other hand, $M_{P}$ seems to be bounded from below and does not approach zero even in the chiral limit. The bound on $M_{P}$ depends on the size of lattices in proportion to $\sim L^{-1}$, and thus this seems to be caused by the finite size effect on $M_{P}$. In order to judge whether the chiral symmetry breaking occurs in this system, we analyze the quantity $R \equiv a^{1 / 2} M_{P} / m_{\mathrm{PCAC}}{ }^{1 / 2}$ (Fig. [).

Because of the finite size effect on $M_{P}, R$ depends clearly on the system size for small quark masses and diverges for $m_{\text {PCAC }} \rightarrow 0$. In this observation, we neglect data with visible finite size effect. Then, we notice the most important point from Fig. 1 ; $M_{P}$ is proportional to $m_{\mathrm{PCAC}}{ }^{1 / 2}$ for $0.35<a m_{\mathrm{PCAC}}<0.65$, but it changes with a different exponent, $M_{P}$ is proportional to $m_{\mathrm{PCAC}}{ }^{\alpha}$ with $\alpha>0.5$, for smaller quark mass region $a m_{\mathrm{PCAC}} \lesssim 0.3$. If the chiral symmetry breaking is realized in the system, $M_{P}$ should be better approximated by $m_{q}{ }^{1 / 2}$ for lighter quarks, which is not acturally the case. This is the first evidence supporting the absence of chiral symmetry breaking in the six-flavor theory.

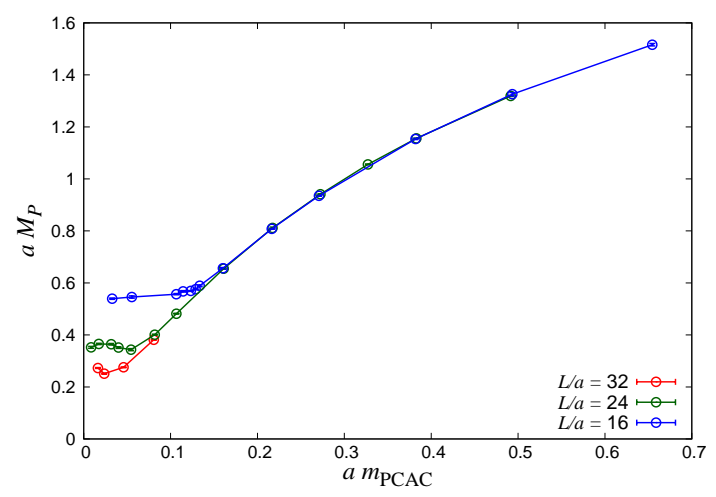

Figure 1: The lightest pseudoscalar meson mass $a M_{P}$ versus $a m_{\mathrm{PCAC}}$ in six-flavor theory.

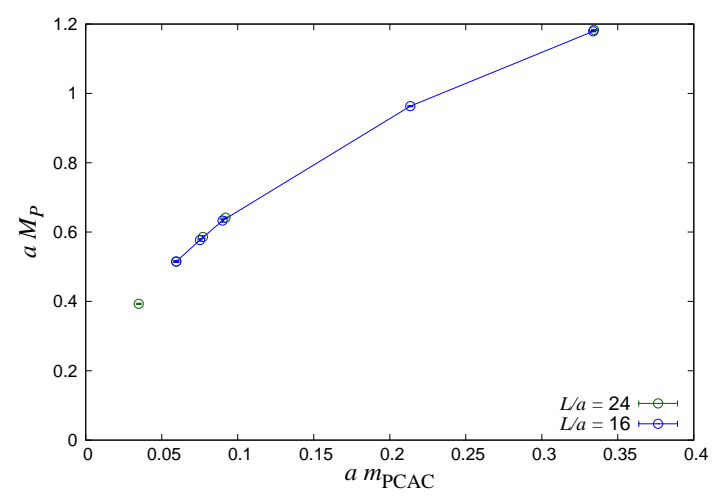

Figure 3: The lightest pseudoscalar meson mass $a M_{P}$ versus $a m_{\mathrm{PCAC}}$ in two-flavor theory.

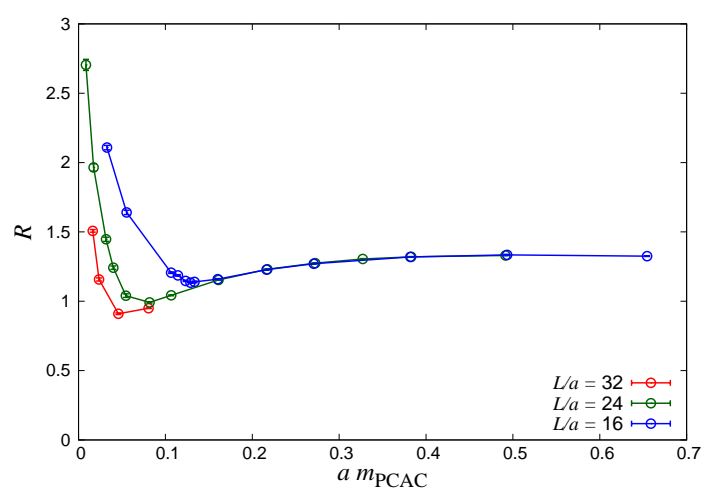

Figure 2: The ratio $R=a^{1 / 2} M_{P} / m_{\mathrm{PCAC}}{ }^{1 / 2}$ versus $a m_{\mathrm{PCAC}}$ in six-flavor theory.

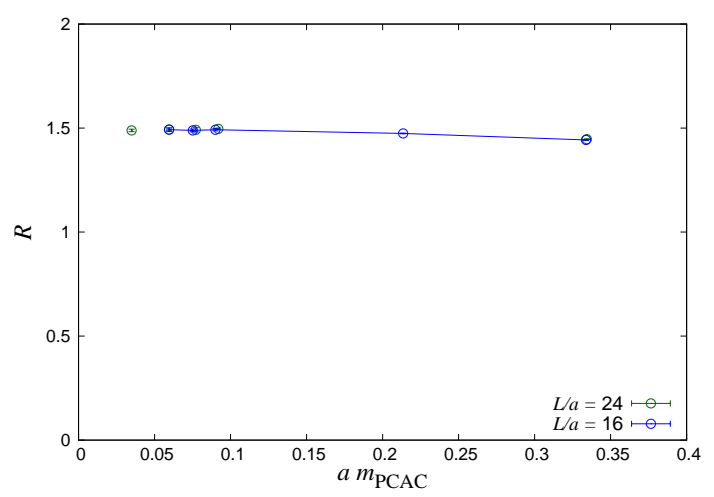

Figure 4: The ratio $R=a^{1 / 2} M_{P} / m_{\mathrm{PCAC}}{ }^{1 / 2}$ versus $a m_{\mathrm{PCAC}}$ in two-flavor theory.

In order to make this evidence more determinate, we compare this result with the result in the two-flavor system. In the simulation of the two-flavor theory, we employ lattices with sizes 
$L / a=16,24$ and the same $\beta(=2.0)$ as in the six-flavor theory. Figure 3 shows the quark mass dependence of $M_{P}$ in two-flavor theory, where no visible finite size effect on $M_{P}$ is seen. Figure $⿴$ shows the quark mass dependence of $R$, where $M_{P}$ is better approximated by $m_{\mathrm{PCAC}}{ }^{1 / 2}$ for smaller $m_{\mathrm{PCAC}}$. This behavior is compatible with the behavior in the system with chiral symmetry breaking and able to be distinguished clearly from the behavior in the case of $N_{f}=6$.

\section{Decay Constant}

Next, we focus on the decay constant of the lightest pseudoscalar meson: $f_{P}$. In the observation of this quantity, we especially pay attention to the behavior of the finite size effect on $f_{P}$. Figure [ shows a sketch of the quark mass and volume dependence of $f_{P}$ which is theoretically expected for the system with the breakdown of chiral symmetry. The important point is that the finite size effect tends to decrease $f_{P}$. This tendency is observed for both the $\varepsilon$-regime [ $\left.\square\right]$ ] and p-regime [ए2]. Therefore, if the finite size effect turns out to increase $f_{P}$, it will give an evidence that chiral symmetry breaking does not occur in the system.

Figure 6 shows the quark mass dependence of $f_{P}$ defined by the equation;

$$
f_{P}=2 \kappa \cdot 2 m_{\mathrm{PCAC}} \sqrt{\frac{2 A^{P P}}{M_{P}}} \frac{1}{\sinh M_{P}},
$$

which is derived from PCAC relation. In eq. (B.]), $A^{P P}$ is the amplitude of the PP correlator

$$
\langle P(t) P(0)\rangle \stackrel{a \ll t \ll T}{\longrightarrow} A^{P P}\left(e^{-M_{P} t}+e^{-M_{P}(T-t)}\right) .
$$

From Fig. $6, f_{P}$ seems to vanish in the chiral limit. When we pay attention to the definition (B.d), however, we notice that this is not necessarily true because of the finite size effect on the pseudoscalar meson mass $M_{P}$, which exists in the denominator of R.H.S. of eq. (B. W) while $m_{\mathrm{PCAC}}$ exists in the numerator. Since $M_{P}$ at a finite volume system is bounded from below at small quark

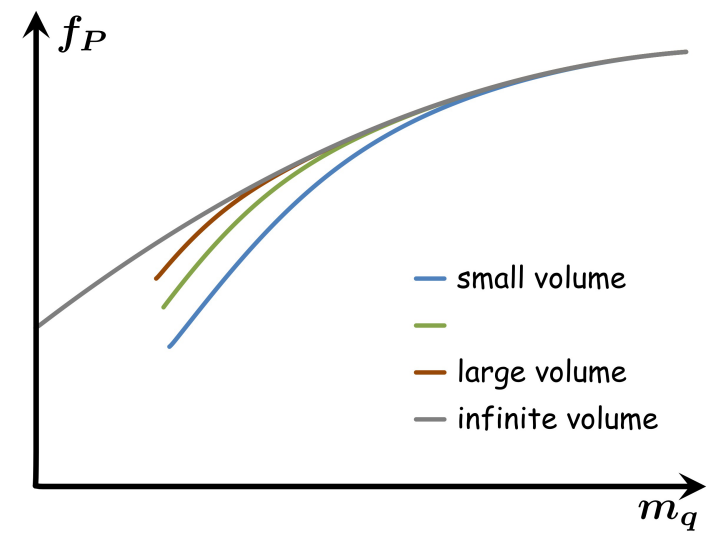

Figure 5: A sketch of quark mass and volume dependence of $f_{P}$ in the system with the breakdown of chiral symmetry. The finite size effect tends to decrease $f_{P}$.

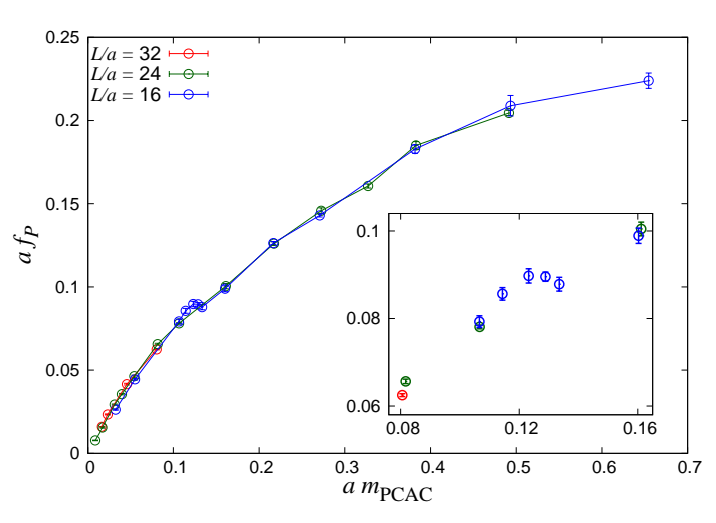

Figure 6: The decay constant $a f_{P}$ versus $a m_{\mathrm{PCAC}}$ in six-flavor theory. The finite size effect seems to increase $f_{P}$ around the region $a m_{\mathrm{PCAC}}=0.12 \sim$ 0.14 . 
mass region and cannot vanish in the chiral limit, $f_{P}$ defined as (B. vanishes even if chiral symmetry breaking occurs in this system. Therefore, the result in the mass region where the finite size effect on $M_{P}$ dominates should be neglected.

Then, we notice that, for the lattice size $L / a=16$, there is the quark mass region $a m_{\mathrm{PCAC}}=$ $0.12 \sim 0.14$ where the finite size effect seems to increase $f_{P}$. The behavior of this finite size effect is opposite to that in the system with chiral symmetry breaking. This is the second signature supporting that the chiral symmetry breaking does not occur in the six-flavor theory.

\section{Chiral Condensate}

Lastly, we discuss the chiral condensate $\langle\bar{\psi} \psi\rangle$, which is the order parameter of chiral symmetry breaking. Since we use the Wilson fermion, however, $\langle\bar{\psi} \psi\rangle$ is dominated by the UV divergence $\sim O\left(a^{-3}\right)$, which does not vanish in the chiral limit. Instead of $\langle\bar{\psi} \psi\rangle$, we focus on the subtracted chiral condensate $\langle\bar{\psi} \psi\rangle_{\text {subt }}$, which is free from such a cubic divergence. The subtracted chiral condensate in the Wilson fermion is calculated by using the Ward-Takahashi identity with respect to the axial-vector current [[13];

$$
\langle\bar{\psi} \psi\rangle_{\text {subt }}=2 m_{\mathrm{PCAC}} \cdot(2 \kappa)^{2} \sum_{n}\langle P(n) P(0)\rangle .
$$

Before discussing the result in the six-flavor theory, we will discuss the utility of the subtracted chiral condensate by analyzing the result in the two-flavor theory. Figure $\square$ shows the quark mass dependence of $\langle\bar{\psi} \psi\rangle_{\text {subt }}$ in the two-flavor theory with two fit lines. Since there is no visible volume dependence on $\langle\bar{\psi} \psi\rangle_{\text {subt }}$, we assume these data do not suffer the finite size effect and results of these fit give the value in the infinite volume system. The dotted line is drown by fitting the linear function

$$
f_{2}\left(x=a m_{\mathrm{PCAC}}\right)=a_{0}+a_{1} x
$$

to the data of $a m_{\mathrm{PCAC}}<0.1$, while the red line is drown also by fitting the quadratic function

$$
f_{3}\left(x=a m_{\mathrm{PCAC}}\right)=b_{0}+b_{1} x+b_{2} x^{2}
$$

to all data. Then, we find

$$
\left.\lim _{m_{\mathrm{PCAC} \rightarrow 0}} a^{3}\langle\bar{\psi} \psi\rangle_{\text {subt }}\right|_{N_{f}=2}=\left\{\begin{array}{l}
0.0230(22) \text { linear fit } \\
0.0185(17) \text { quadratic fit }
\end{array} .\right.
$$

Both of these values indicate that the chiral limit of $\langle\bar{\psi} \psi\rangle_{\text {subt }}$ is nonzero and much smaller than $a^{-3}$ so we assume the chirally extrapolated value of $\langle\bar{\psi} \psi\rangle_{\text {subt }}$ is free from the UV divergence. Therefore, we calculate $\langle\bar{\psi} \psi\rangle_{\text {subt }}$ also in the six-flavor theory.

Figure $\mathbb{8}$ shows the quark mass dependence of $\langle\bar{\psi} \psi\rangle_{\text {subt }}$ in the six-flavor theory. We perform the chiral extrapolation also for this result to judge whether the extrapolated value is consistent with zero within available precision, i.e. whether the result supports two signatures gained from the results of $M_{P}$ and $f_{P}$, which indicate that chiral symmetry breaking does not occur in this system. There is no visible volume dependence on calculated values of $\langle\bar{\psi} \psi\rangle_{\text {subt }}$. Therefore we assume these data suffer few finite size effect so that we can use the value at the lightest quark mass 


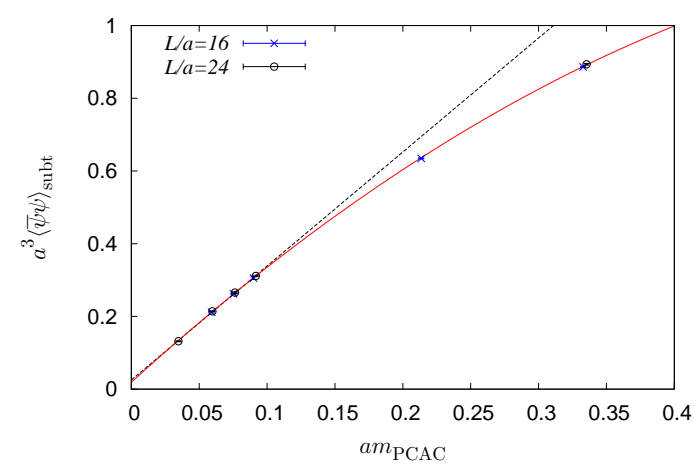

Figure 7: The subtracted chiral condensate $\langle\bar{\psi} \psi\rangle_{\text {subt }}$ versus $a_{\mathrm{PCAC}}$ in two-flavor theory with linear(dotted line) and quadratic(red line) fit lines.

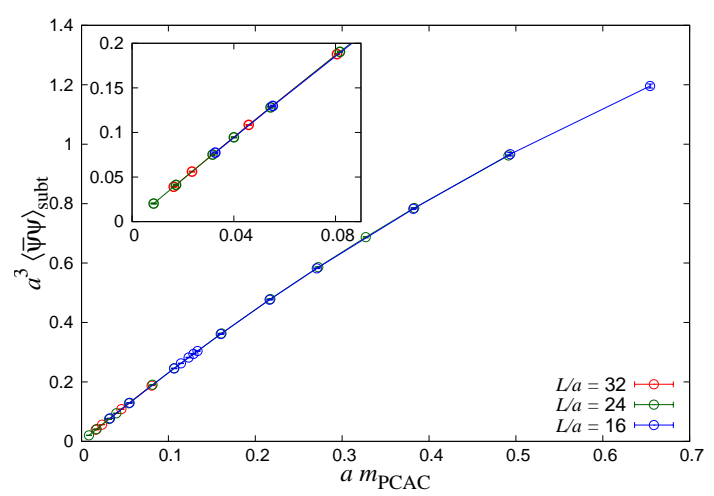

Figure 8: The subtracted chiral condensate

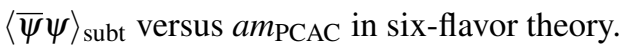

as one of target data to be fit. We introduce the result of the quadratic fit (4.3) using following two data sets;

(S1): data with $a m_{\mathrm{PCAC}} \leq 0.2$,

(S2): data with $a m_{\mathrm{PCAC}} \leq 0.1$

The fit gives

$$
\left.\lim _{m_{\mathrm{PCAC}} \rightarrow 0} a^{3}\langle\bar{\psi} \psi\rangle_{\text {subt }}\right|_{N_{f}=6}=\left\{\begin{array}{rl}
-0.00001(27) & \text { data set : S1 } \\
0.00012(41) & \text { data set : S2 }
\end{array},\right.
$$

which are vanishing extrapolated values within available precision. This is the third signature supporting the absence of the breakdown of chiral symmetry.

Meanwhile, we also perform the power type fit;

$$
f_{\text {pow }}\left(x=a m_{\mathrm{PCAC}}\right)=c_{1} x+c_{2} x^{\alpha},
$$

which is expected for small quark masses in the system with IR-fixed point. If the system is conformal in the infrared and the hyper-scaling hypothesis is valid, $\alpha$ is equal to $\left(3-\gamma_{\star}\right) /\left(1+\gamma_{\star}\right)$, where $\gamma_{\star}$ denotes the mass anomalous dimension at the IR-fixed point [प4]]. As a result, we obtain $\alpha=1.76(66)$ for the data set $\mathbf{S 2}$. This indicates

$$
0.17 \leq \gamma_{\star} \leq 0.90
$$

which is not inconsistent with that found in the study of the running coupling constant defined in the Schrödinger functional scheme [ए5]];

$$
0.26 \leq \gamma_{\star}, \mathrm{SF} \leq 0.74
$$

\section{Summary}

We investigated the chiral behavior of the two-color QCD with six fundamental Dirac fermions by analyzing the quark mass dependence and the volume dependence of some quantities with very 
careful attention to the finite size effects on these quantities. Since we observe that the presented three quantities behave oppositely to the prediction from the theory with chiral symmetry breaking, we conclude the breakdown of chiral symmetry does not occur in this gauge system.

More detailed and more quantitative discussions are summarized in the full paper [ए0]]. The same conclusion is obtained in the calculation of the SF running coupling constant reported in the companion paper [15]].

\section{Acknowledgments}

The numerical simulations were carried out on the computer system $\varphi$ at Nagoya University, and the servers equipped with GPU cards at High Energy Accelerator Research Organization (KEK). This work is supported partly by JSPS Grands-in-Aid for Scientific Research 20540261, $22224003,22740183,23740177$.

\section{References}

[1] S. Catterall and F. Sannino, Phys. Rev. D76 (2007) 034504, arXiv: 0705.1664 [hep-lat ].

[2] A. J. Hietanen, K. Rummukainen, and K. Tuominen, Phys. Rev D80 (2009) 094504, arXiv:0904.0864 [hep-lat].

[3] T. DeGrand, Y. Shamir, and B. Svetitsky, Phys. Rev. D83 (2011) 074507, arXiv: 1102.2843 [hep-lat].

[4] L. Del Debbio, B. Lucini, A. Patella, C. Pica, and A. Rago, Phys. Rev. D82 (2010) 014509 , arXiv:1004.3197 [hep-lat].

[5] T. van Ritbergen, J. Vermaseren, and S. Larin, Phys. Lett. B400 (1997) 379-384, arXiv:hep-ph/9701390 [hep-ph].

[6] Y. Iwasaki, K. Kanaya, S. Kaya, S. Sakai, and T. Yoshie, Phys. Rev. D69 (2004) 014507, arXiv:hep-lat/0309159 [hep-lat].

[7] F. Bursa, L. Del Debbio, L. Keegan, C. Pica, and T. Pickup, Phys. Lett. B696 (2011) 374, arXiv:1007.3067 [hep-ph].

[8] H. Ohki, et al., PoS LATTICE 2010 (2010) 066, arXiv:1011.0373 [hep-lat ] .

[9] T. Karavirta, J. Rantaharju, K. Rummukainen, and K. Tuominen, JHEP 1205 (2012) 003 , arXiv:1111.4104 [hep-lat].

[10] M. Hayakawa, K.-I. Ishikawa, S. Takeda, M. Tomii, and N. Yamada, arXiv:1307.6696 [hep-lat].

[11] S. Aoki and H. Fukaya, Phys. Rev. D84 (2011) 014501, arXiv:1105.1606 [hep-lat].

[12] G. Colangelo, S. Durr, and C. Haefeli, Nucl. Phys. B721 (2005) 136-174, arXiv:hep-lat/0503014 [hep-lat].

[13] M. Bochicchio, L. Maiani, G. Martinelli, G. C. Rossi, and M. Testa, Nucl. Phys. B262 (1985) 331.

[14] L. Del Debbio and R. Zwicky, Phys. Rev. D82 (2010) 014502, arXiv:1005.2371 [hep-lat].

[15] M. Hayakawa, K.-I. Ishikawa, S. Takeda, and N. Yamada, arXiv:1307.6997 [hep-lat ]. 